\title{
Design of the HTS Fusion Conductors for TF and CS Coils
}

\author{
Nikolay Bykovsky, Davide Uglietti, Rainer Wesche, and Pierluigi Bruzzone
}

\begin{abstract}
The main electrical and mechanical requirements for the LTS fusion conductors of DEMO are retained as a starting point for the development of HTS fusion cables. Based on the HTS coated conductor technology, a flat cable design was proposed by CRPP Swiss Plasma Center (SPC) using the strands made of twisted stack of tapes soldered into copper profiles. Analytical modeling of the cable geometry is developed and presented in this work. The model was used to estimate various properties of cable. Addressing the issue of bending strain and related performance degradation, an optimization model of the cable properties was built, which allows to best fulfill the cable requirements. Design options are developed for both toroidal field (TF) coils operating at $63 \mathrm{kA}$ and central solenoid (CS) coils operating at $50 \mathrm{kA}$. Paying attention to the DC and pulsed operation of the TF and CS coils, proposals for the design of the forced-flow HTS conductors are reported and discussed for each type of the coils.
\end{abstract}

Index Terms-HTS fusion cables, TF and CS coils.

\section{INTRODUCTION}

A NOVEL DESIGN of the high current HTS strand for fusion cables was proposed recently at SPC [1]. After the first test trials, the idea of a stacking, twisting and soldering of the HTS tapes and two copper profiles converged to the strand's design presented in Fig. 1. Poor bending properties of the first samples were initially discovered and soon effectively improved by using the preliminary annealed copper profiles [2]. This improvement allowed us to propose a first suitable $60 \mathrm{kA} / 12$ T HTS cable design based on such strands. Sketch of the cable (copper cored Rutherford design) and definition of its main geometrical parameters are given in Fig. 2.

The manufacture of two cable prototypes started in the middle of 2014 and was successfully finished in the beginning of 2015. In June 2015 the sample was tested in the EDIPO test facility at SPC, including $I_{c}, T_{c s}$, AC loss and cycling measurements. The details of the manufacturing process and test results will be published in [3]. Comparing the assessed and measured DC performance of the cables, the tape transport properties were fully retained in the prototypes. At the same time, two issues were discovered: around 3\% degradation of $I_{c}$ for the both cables after the cycling test (1000 cycles of the current from 0 to $50 \mathrm{kA}$ at $12 \mathrm{~T}$ ) and relatively high $\mathrm{AC}$ loss-from 2 to 10 times larger than in the ITER TF conductors-which was mainly related to inter-strand currents.

Manuscript received October 14, 2015; accepted November 28, 2015. Date of publication December 17, 2015; date of current version January 11, 2016.

The authors are with the EPFL Swiss Plasma Center, Paul Scherrer Institute, Villigen 5232, Switzerland (e-mail: nikolay.bykovsky@psi.ch).

Color versions of one or more of the figures in this paper are available online at http://ieeexplore.ieee.org.

Digital Object Identifier 10.1109/TASC.2015.2509178

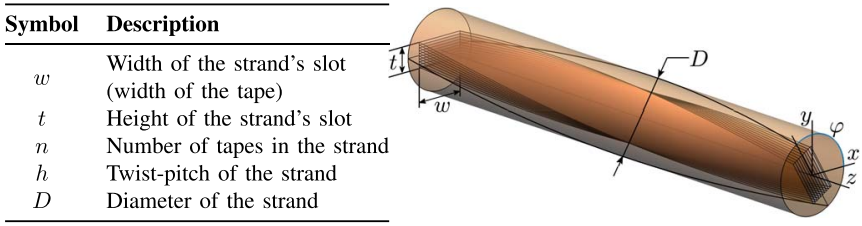

Fig. 1. Sketch of the strand and definition of the geometrical parameters.

\begin{tabular}{cl}
\hline Symbol & Description \\
\hline$N$ & Number of strands in the cable \\
$g$ & Thickness of the cable core \\
$f$ & Width of the flat part of core \\
$\theta$ & Twist-angle of the cable \\
$L$ & Twist-pitch of the cable \\
\hline
\end{tabular}

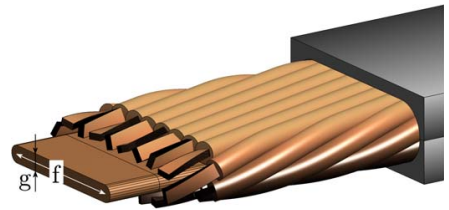

Fig. 2. Sketch of the cable and definition of the geometrical parameters.

In order to further optimize the strand design and investigate reasons of the degradation during the cycling test, various electromechanical tests (twisting, bending and transverse pressure) were performed with the strands of various tapes and profile geometry [4]. Higher current density of the strand, lower AC loss and improved mechanical strength against transverse pressure can be achieved with a square stack of $3 \mathrm{~mm}$-width tapes.

The goal of the cable design is to fulfill appropriate requirements (i.e. operating current, temperature margin, minimal bending radius, AC loss etc) by using an initially selected strand design. In this work, we present a general discussion over the copper cored Rutherford cable design. An analytical model of the cable geometry is presented and implemented for the estimation of various mechanical and electrical properties of the cable. Optimization methods were applied in order to best fulfill declared requirements of TF and CS coils of fusion magnets. As a result, the cable design proposals for these coils are obtained, presented and discussed in the last section.

\section{Geometry of the CABle}

Five geometrical parameters are used to describe the cored Rutherford geometry (see Fig. 2), but only three of them are independent. Two necessary link equations can be written by considering the flat pattern of the cable core and the contact line between the strand and the core, which are presented in Fig. 3. The pattern is divided in four sections corresponding to the two flat and two rounded regions of the core cross section. Helical shape of the strand at the cable edges transforms to a straight line in a flat pattern view. As a result, twist-pitch of the cable $L$ can be written in a two ways:

$$
\begin{aligned}
L & =(2 f+\pi(g+D)) \cot \theta \\
L & =N \frac{D}{\sin \theta} .
\end{aligned}
$$




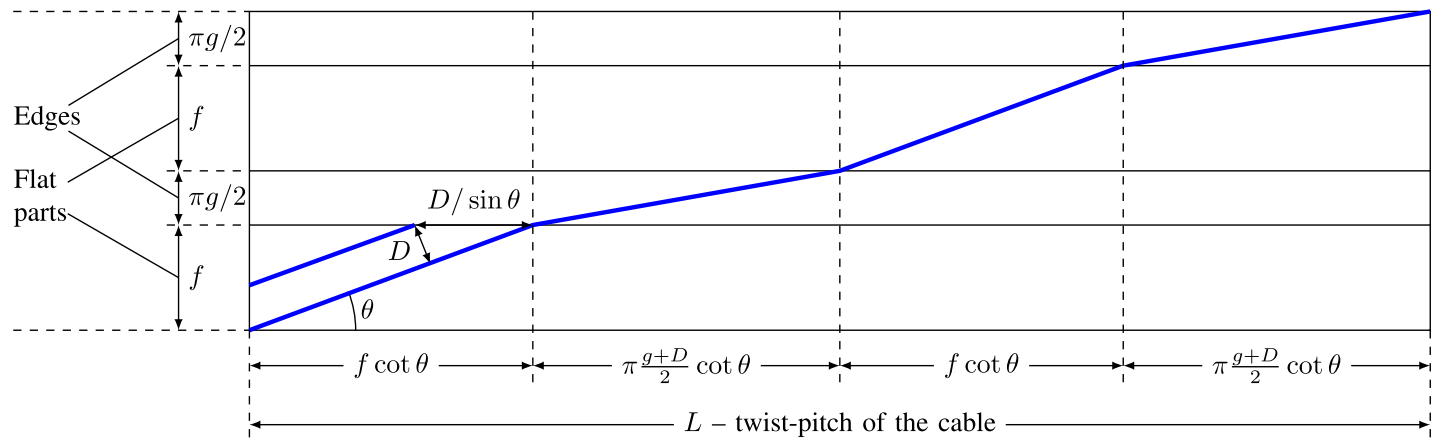

Fig. 3. Flat pattern of the cable core. Blue line corresponds to contact between the strand and cable core.

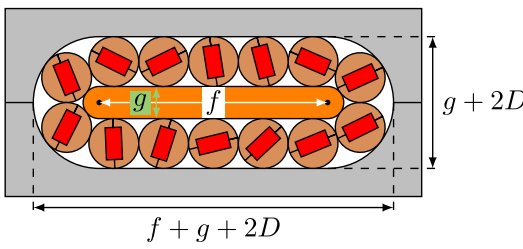

Fig. 4. Cross section of the 14-strand cable.

Considering $(N, g, L)$ as independent parameters, one can express $f$ and $\theta$ from (1) as follows:

$$
\begin{aligned}
\theta & =\arcsin \frac{N D}{L} \\
f & =\frac{\pi}{2}\left(g_{c}-g\right) \\
g_{c} & =\left(\frac{N}{\pi \cos \theta}-1\right) D .
\end{aligned}
$$

The cable core becomes round $(f=0)$ by setting $g=g_{c}$, i.e., the new notation $g_{c}$ corresponds to the diameter of the round core. This is the maximum value for $g: 0 \leqslant g \leqslant g_{c}$.

In the model the strands are assumed to contact each other at equatorial points of the cross section. This is a valid assumption for the flat parts of the core and is a good approximation for the edges if $g \gtrsim D$. In case of $g \lesssim D$ the model is less accurate: at the cable's edge the strands contact each other at the points lower than the strand's equator. This effect was studied numerically and for $N>14$ the analytical result (2) is lower than a more precise numerical one by less than $4 \%$.

An example of the cross section of 14-strand cable is presented in Fig. 4. According to the drawing, the cable space $S_{\text {cable }}$ and the total copper cross section $S_{\mathrm{cu}}$ can be expressed as:

$$
\begin{aligned}
S_{\text {cable }} & =f(g+2 D)+\pi(g / 2+D)^{2} \\
S_{\mathrm{cu}} & =f g+\pi g^{2} / 4+N\left(\pi D^{2} / 4-w\left(t-n d_{\mathrm{cu}}\right)\right)
\end{aligned}
$$

where $d_{\mathrm{cu}}$ is the thickness of the copper layers in a single tape (typically $40 \mu \mathrm{m}$ ).

The space available for the coolant flow (i.e., cross section of the cable voids) $S_{\text {flow }}$ and wetted perimeter of the cable $P_{\text {wetted }}$ are

$$
\begin{aligned}
S_{\text {flow }} & =2 f D+\pi g D+\pi D^{2}(1-N / 4) \\
P_{\text {wetted }} & =4 f+2 \pi g+\pi D(N+2)
\end{aligned}
$$

which allows finally to calculate the hydraulic diameter $D_{h}$ and the void fraction of the cable cross section: $D_{h}=4 S_{\text {flow }} / P_{\text {wetted, }}$,
$\mathrm{vf}=S_{\text {flow }} / S_{\text {cable }}$ - the geometrical parameters used in the hydraulic calculations.

\section{Mechanical Strain During Manufacturing}

Due to the transposition requirement, bending deformations must be applied to strands during the fabrication of the cable in order to achieve required twist-pitch of the cable. An effective bending radius of the strand at the cable edge can be written as [2]:

$$
R=\frac{g+D}{2 \sin ^{2} \theta} .
$$

As the strain distribution in bent twisted strand is not uniform, critical bending radius of the strand $R_{c}$ will be used as integral measure. $R_{c}$ is typically around $200 \mathrm{~mm}$ for $5 \% I_{c}$ degradation criterion (see [4] for the full details).

One would like to select the twist pitch at cable level as short as possible (for example to limit AC losses), but short twistpitch of the cable $L$ leads to very low bending radius $R$, which in turn can not be shorter than $R_{c}$, otherwise the critical current is strongly reduced. The minimal permissible twist-pitch of the cable $L_{\min }$ corresponds to the lowest strand's bending radius at the cable edge $\left(R=R_{c}\right)$ and can be estimated from (1) and (5) as:

$$
L_{\text {min }}=N D \sqrt{\frac{2 R_{c}}{g+D}} .
$$

Thus, the mechanical properties of the strand impose the following restriction for the twist-pitch of the cable: $L>L_{\min }$. Presence of the central core in the cable design (i.e. $g>0$ ) allows to lower $L_{\min }$ and to make smaller values of $L$ available - the key reason for including this design feature.

For the coil fabrication stage, the cable should be additionally bent (in a plane perpendicular to the flat part of the core) in order to reach a specified shape. Assuming the position of a neutral axis in the center of the cable core, a peak bending strain will occur in the outer strands layer (relative to the bending center of the cable) at the corner of the stack of tapes. As the bending of the cable at radius $r$ will result in effective strand's bending radius $r / \cos ^{2} \theta$, the total peak strain one can estimate as:

$$
\varepsilon_{\text {peak }}=\frac{d}{2 R}+\frac{(g+D+d) \cos ^{2} \theta}{2 r}
$$

where $d$ is the diagonal of the stack: $d=\sqrt{w^{2}+t^{2}}$. 
Maximal bending strain of the strand is $d /\left(2 R_{c}\right)$, which allows finally to estimate the minimum bending radius of the cable:

$$
r_{c}=R_{c}\left(1+\frac{g+D}{d}\right) \frac{L^{2}-N^{2} D^{2}}{L^{2}-L_{\min }^{2}} .
$$

Note that $r_{c}=\infty$ by setting $L=L_{\text {min }}$, which means that such cable cannot be bent otherwise the critical strain on the ceramic is exceeded. According to the coil geometry, the proper design of the cable should meet the following condition: $r_{c}<r_{\text {coil }}^{\mathrm{min}}$.

\section{Electrical Properties}

\section{A. $T_{c s}$ Calculation}

The critical current of the cable $I_{c}$ is affected by the self-field effect even at high background magnetic fields, but this effect is almost completely compensated by the angular dependence of the tape's critical current $I_{c}^{t}$ at least in the operating conditions from $40 \mathrm{kA}$ to $70 \mathrm{kA}$ and from $8 \mathrm{~T}$ to $12 \mathrm{~T}$ [3]. Eventually, a simple and reliable approach to estimate $I_{c}$ is to sum up $I_{c}^{t}$ corresponding to the perpendicular field component. Considering identical tapes, one can write: $I_{c}(B, T)=N n I_{c_{\perp}}^{\text {tape }}(B, T)$. Therefore, for the cable with operating current $I_{\text {op }}$ at external magnetic field $B$, the current sharing temperature $T_{c s}$ can be obtained from the following equation:

$$
\begin{aligned}
I_{\mathrm{op}} & =N n w j_{c_{\perp}}\left(B, T_{c s}\right) \\
j_{c_{\perp}}(B, T) & =A \frac{B_{0}(T)^{\beta}}{B}\left(\frac{B}{B_{0}(T)}\right)^{p}\left(1-\frac{B}{B_{0}(T)}\right)^{q} \\
B_{0}(T) & =B_{0}\left(1-\frac{T}{T_{0}}\right)^{\alpha}
\end{aligned}
$$

where $A=0.9 \mathrm{AT}^{1-\beta} / \mathrm{mm}, \alpha=3.48, \beta=1.61, p=0.54$, $q=2.82, B_{0}=170.78 \mathrm{~T}, T_{0}=138.91 \mathrm{~K}$. These parameters are obtained from the $I_{C_{\perp}}^{\text {tape }}(B, T)$ data of the SuperOx tapes for the $1 \mu \mathrm{V} / \mathrm{cm}$ criterion (data from the manufacturer). The scaling law $j_{c_{\perp}}(B, T)$ has a high accuracy at temperatures lower than $40 \mathrm{~K}$ and field range from $8 \mathrm{~T}$ to $12 \mathrm{~T}$.

Since the $I_{c}(B, T)$ characterization of the HTS tapes is typically performed at $1 \mu \mathrm{V} / \mathrm{cm}$, this calculation is able to estimate $T_{c s}$ only for that criterion, while for the LTS fusion cables $T_{c s}$ is usually defined at $0.1 \mu \mathrm{V} / \mathrm{cm}$. The difference between the $T_{c s}$ values defined at these criteria depends on the $m$-value of the $T-E$ transition. In accordance with the test results of the HTS cable prototype [3], this difference can be estimated as $\approx 2 \mathrm{~K}$ at $12 \mathrm{~T}$ and for operating currents between $50 \mathrm{kA}$ to $60 \mathrm{kA}$.

\section{B. Current Densities of the Cable}

Requirements for the cable's compactness and safety during the quench can be expressed in terms of the cable and copper current densities at operating conditions: $j_{\text {cable }}=I_{\mathrm{op}} / S_{\text {cable }}$ and $j_{\mathrm{cu}}=I_{\mathrm{op}} / S_{\mathrm{cu}}\left(j_{\mathrm{cu}}\right.$ corresponds to the quench situation, when $I_{\mathrm{op}}$ flows only in the copper cross section of the cable). Appropriate cross sections can be calculated from (3). The typical limits for fusion cables: $j_{\text {cable }} \gtrsim 50 \mathrm{~A} / \mathrm{mm}^{2}$ including cooling

\begin{tabular}{|c|c|c|c|c|c|c|c|}
\hline \multirow{2}{*}{$\begin{array}{l}\text { Coil } \\
\text { type }\end{array}$} & \multirow{2}{*}{$\begin{array}{l}I_{\mathrm{op}} \\
\mathrm{kA}\end{array}$} & \multicolumn{4}{|c|}{ Bottom limits } & \multicolumn{2}{|c|}{ Top limits } \\
\hline & & $\begin{array}{c}T_{c s}^{*} \\
\mathrm{~K}\end{array}$ & $\begin{array}{c}j_{\text {cable }} \\
\mathrm{A} / \mathrm{mm}^{2}\end{array}$ & $\begin{array}{l}r_{\text {coil }}^{\min } \\
\mathrm{m}\end{array}$ & $\begin{array}{c}p_{c} \\
\mathrm{MPa}\end{array}$ & $\begin{array}{c}j_{\mathrm{cu}} \\
\mathrm{A} / \mathrm{mm}^{2}\end{array}$ & $\begin{array}{c}Q_{\text {total }}^{* *} \\
\mathrm{~J} /(\mathrm{m} \cdot \text { cycle })\end{array}$ \\
\hline $\mathrm{TF}$ & 63 & 7.5 & 50 & 3.0 & 15 & 100 & 15 \\
\hline $\mathrm{CS}$ & 50 & 8.5 & 50 & 1.9 & 15 & 120 & 5 \\
\hline
\end{tabular}
channels; $j_{\text {cu }} \lesssim 100 \mathrm{~A} / \mathrm{mm}^{2}$.
TABLE I

CABle ReQuirements FOR TF AND CS Coils at $B=12 \mathrm{~T}$

\section{Transverse Load During the Cable Operation}

Due to the acting Lorentz forces in the cable, another important electromechanical requirement at the strand level is the stability against the transverse pressure. Discussion of this phenomenon for various HTS strands is published recently in [4]. According to the Lorentz force: $p=2 I_{\mathrm{op}} B /(N D)<p_{c}$, where $B$ is the background magnetic field and $p_{c}$ is the pressure limit, which depends on the strand design and is in the range from $20 \mathrm{MPa}$ to $35 \mathrm{MPa}$.

\section{AC Loss Estimation}

Finally, in order to assess total AC losses of the cable in the alternating magnetic field, the measured value on HTS cable prototype of around $20 \mathrm{~J} /(\mathrm{m} \cdot$ cycle $)$ at $\nu=0.1 \mathrm{~Hz}$ and $B_{a}=$ $0.2 \mathrm{~T}$ was used. AC field is applied perpendicular to the wide side of the cable. This value can be rescaled for new strand's and cable's geometries according to [5], [6].

\section{Design Proposals}

In summary, properties of the HTS tape and 8 geometrical parameters -5 for the strand and 3 for the cable - are required to propose the cable design. In order to best fulfill the various cable requirements, an optimization model was built, where the cable requirements are divided in two types: the first type imposes the bottom limit on the cable property, and the second one imposes the upper limit. Using the dimensionless factors $\xi$, each type of the requirements can be written as:

$$
\xi_{\mathrm{b}}=\frac{\text { bottom limit }}{\text { cable property }}<1 \quad \xi_{\mathrm{u}}=\frac{\text { cable property }}{\text { upper limit }}<1 .
$$

For instance, the requirements for the bending radius of the cable $r_{\text {coil }}^{\min }$, strand's transverse pressure $p_{c}$, copper current density $j_{\text {cu }}$ and total AC energy loss of the cable $Q_{\text {total }}$ are the first type, while the requirements for the engineering current density of the cable $j_{\text {cable }}$ and current sharing temperature $T_{c s}$ are the second type.

These factors allow to consider the optimization problem from a single point of view: fulfillment of the requirement means the minimization of the appropriate factor. If a set of $m$ cable requirements is considered at the same moment, the optimization problem of the cable design can be formulated as a search of such cable geometry that minimizes the sum of the appropriate factors $\xi_{i}$ :

$$
\min \sum_{i=1}^{m} \xi_{i}: \quad \xi_{i}<1, \quad i=1, \ldots, m .
$$


TABLE II

Cable Design Propos Als For TF AND CS CoILS

\begin{tabular}{|c|c|c|c|c|c|c|c|c|c|c|c|c|c|c|c|c|}
\hline \multirow{2}{*}{$\begin{array}{l}\text { Coil } \\
\text { type }\end{array}$} & \multirow{2}{*}{$\begin{array}{l}\text { Sketch of the } \\
\text { cross-section }\end{array}$} & \multicolumn{8}{|c|}{ Parameters } & \multicolumn{7}{|c|}{ Properties at $12 \mathrm{~T}$} \\
\hline & & $\begin{array}{c}w \\
\mathrm{~mm}\end{array}$ & $\begin{array}{c}t \\
\mathrm{~mm}\end{array}$ & $\begin{array}{l}n \\
-\end{array}$ & $\begin{array}{c}h \\
\mathrm{~mm}\end{array}$ & $\begin{array}{c}D \\
\mathrm{~mm}\end{array}$ & $\begin{array}{l}N \\
-\end{array}$ & $\begin{array}{c}g \\
\mathrm{~mm}\end{array}$ & $\begin{array}{l}L \\
\mathrm{~m}\end{array}$ & $\begin{array}{c}T_{c s}^{*} \\
\mathrm{~K}\end{array}$ & $\begin{array}{c}I_{c}(5 \mathrm{~K}) \\
\mathrm{kA}\end{array}$ & $\begin{array}{l}r_{c} \\
\mathrm{~m}\end{array}$ & $\begin{array}{c}p \\
\mathrm{MPa}\end{array}$ & $\begin{array}{r}j_{\text {cabl }} \\
\mathrm{A} /\end{array}$ & $\mathrm{nm}^{2}$ & $\begin{array}{c}Q_{\text {total }}^{* *} \\
\mathrm{~J} /(\mathrm{m} \cdot \text { cycle })\end{array}$ \\
\hline $\mathrm{TF}$ & 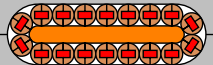 & 4 & 2.1 & 20 & 300 & 6.3 & 18 & 5 & 0.9 & 7.7 & 69.1 & 2.5 & 13.3 & 67 & 92 & 12.4 \\
\hline $\mathrm{CS}$ & $-8(5)(5)$ & 3 & 3.0 & 28 & 250 & 6.0 & 14 & 5 & 0.7 & 8.5 & 56.4 & 1.8 & 14.3 & 77 & 108 & 3.4 \\
\hline
\end{tabular}

${ }^{*}$ at $1 \mu \mathrm{V} / \mathrm{cm}$ criterion; ${ }^{* *}$ at $\nu=0.1 \mathrm{~Hz}, B_{a}=0.2 \mathrm{~T}$.

Besides that, there are two physical constraints over the strand's parameters: (1) the height of the strand's slot should be enough to hold the tapes, i.e. $t>n d_{t}$, where $d_{t}$ is the tape's thickness; (2) strand's diameter must be higher than the diagonal of the stack, i.e. $D>\sqrt{w^{2}+t^{2}}$. Tolerances for these constraints were chosen as $0.1 \mathrm{~mm}$ and $1.73 \mathrm{~mm}$ respectively.

In order to solve the problem, a constrained multivariable optimization solver (namely, fmincon function) from the MATLAB Optimization Toolbox was used.

Six cable requirements, used for the cable design optimization, are presented in Table I. The TF and CS coils are assumed to operate at $63 \mathrm{kA}$ in a DC current mode and $50 \mathrm{kA}$ in a pulsed current mode respectively, which nearly correspond to the reference DEMO design. Chosen $T_{c s}$ requirements-7.5 K and $8.5 \mathrm{~K}$-lead to a temperature margin of about $2.5 \mathrm{~K}$ and $3.5 \mathrm{~K}$ for $1 \mu \mathrm{V} / \mathrm{cm}$ criterion. According to the previous chapter, it roughly corresponds to appropriate acceptance values of ITER TF and CS coils- $-0.8 \mathrm{~K}$ and $1.5 \mathrm{~K}$. The requirements for minimum bending radius $r_{\text {coil }}^{\min }$ of $3.0 \mathrm{~m}$ and $1.9 \mathrm{~m}$ correspond to the possible geometry of DEMO TF and CS coils respectively. Requirement for the total AC losses $Q_{\text {total }}$ is relatively weak for the TF coil due to DC operation. For the CS coil, $5 \mathrm{~J} /(\mathrm{m} \cdot$ cycle $)$ was taken as an average value for results obtained on Japan LTS cables for ITER CS coil, which fall in the range from $3 \mathrm{~J} /(\mathrm{m} \cdot$ cycle $)$ to $7 \mathrm{~J} /(\mathrm{m} \cdot$ cycle $)$ at $\nu=0.1 \mathrm{~Hz}$ and $B_{a}=0.2 \mathrm{~T}$. Due to a shorter quench time constant of CS coil compared to the TF coil, the top limit for $j_{\mathrm{cu}}$ in CS coil is slightly higher than in TF coil. The operating current density $j_{\text {cable }}$ should be $>50 \mathrm{~A} / \mathrm{mm}^{2}$ for both type of cables. Strand's transverse pressure limit was conservatively set up as $15 \mathrm{MPa}$.

Two design solutions for TF and CS coils were obtained as a result of the optimization. Summary of the conductors parameters and properties together with a sketch of the cross section are given in Table II. Geometry of the copper profiles of the strand (see the sketch of the cables' cross section in Table II) was chosen according to the results recently presented in [4]. These profile geometry is stronger against transverse Lorentz force than the one first introduced. All the requirements mentioned in Table I are fulfilled in the presented cable designs. Lower number of strands in the cable, smaller total width and shorter twist-pitch of the strand and cable allowed to effectively decrease the total AC losses for the CS cable design.
Comparing the critical current of the conductors with appropriate operating current of the coil, one may notice that cables operate at around $\approx 90 \%$ of its full current capacity, while the corresponding value for LTS fusion cables is only around $\approx 50 \%$. This is a direct consequence of the much weaker temperature dependence of the critical current of ReBCO materials compared to LTS (see detailed discussion in [3]). As a result, the HTS cables would require about half the amount of superconductors than LTS ones with the same $T_{c s}$. While the HTS materials are roughly 10 times more expensive, the price ratio between the HTS and LTS cables can be reduced down to 5. Further reduction is expected by the continued improvement of critical current.

\section{CONCLUSION}

Analytical model of the cored Rutherford cable geometry is presented and used for the estimation of the main properties of the proposed HTS cable design. Various requirements on the cable properties corresponding to the main aspects of the TF and CS coils of fusion machines were taken into account during the optimization process of the cable design. The obtained design solutions fulfill all the declared requirements.

In order to verify the validity of the calculated cable properties, fabrication and test of the new HTS cable prototype, based on the design for the CS coil, is planned at EPFL Swiss Plasma Center.

\section{REFERENCES}

[1] D. Uglietti, R. Wesche, and P. Bruzzone, "Fabrication trials of round strands composed of coated conductor tapes," IEEE Trans. Appl. Supercond., vol. 23, no. 3, Jun. 2013, Art. ID 4802104.

[2] N. Bykovsky, D. Uglietti, R. Wesche, and P. Bruzzone, "Strain management in HTS high current cables," IEEE Trans. Appl. Supercond., vol. 25, no. 3, Jun. 2015, Art. ID 4800304.

[3] D. Uglietti et al., "Test of $60 \mathrm{kA}$ coated conductor cable prototypes for fusion magnets," Supercond. Sci. Technol., vol. 28, no. 12, Dec. 1, 2015, Art. ID 124005.

[4] N. Bykovsky, D. Uglietti, R. Wesche, and P. Bruzzone, "Design optimization of round strands made by twisted stacks of tapes," to be published.

[5] A. Campbell, "A general treatment of losses in multifilamentary superconductors," Cryogenics, vol. 22, no. 1, pp. 3-16, Jan. 1982.

[6] V. E. Sytnikov, G. G. Svalov, S. G. Akopov, and I. B. Peshkov, "Coupling losses in superconducting transposed conductors located in changing magnetic fields," Cryogenics, vol. 29, no. 9, pp. 926-930, Sep. 1989. 